Irish Math. Soc. Bulletin

Number 68, Winter 2011, 39-54

ISSN 0791-5578

\title{
QUADRATIC MONOMIAL ALGEBRAS AND THEIR COHOMOLOGY
}

\author{
DAVID O'KEEFFE
}

\begin{abstract}
The aim of this note is to discuss and highlight the use of projective modules and projective resolutions in homological algebra. Using a minimal projective resolution by Sköldberg in [8], we describe the calculation of the cohomology groups for the class of quadratic monomial algebras. The cohomology groups of an associative algebra are invariants of an algebra and provide a fundamental description of the structure of the algebra.
\end{abstract}

\section{INTRODUCTION}

In a forthcoming paper with Emil Sköldberg, the cohomology groups and cohomology ring structure are explicitly described for a particular class of associative algebras: the class of quadratic monomial algebras. Until recently, little was known about the multiplicative structure of the Hochschild cohomology ring for most classes of associative algebras. During the last decade or so, more light has been shed on this topic, with several papers published regarding the structure of the Hochschild cohomology rings for various classes of associative algebras, see for instance [1] and [2] . In [2], Claude Cibils computes the cohomology groups and ring structure for the class of radical square zero algebras. The aforementioned algebras are a subclass of algebras considered in this paper.

\section{QUIVERS AND QUADRATIC MONOMIAL ALGEBRAS}

In this section we begin by recapitulating the following definition of a quiver and from there we will present the main objects of interest in this article, namely the class of quadratic monomial algebras.

2010 Mathematics Subject Classification. 16E40.

Key words and phrases. Quadratic Monomial Algebras, Hochschild cohomology.

Received 20-9-2011; revised 1-3-2012. 
Definition 1. A quiver $\Delta=\left(\Delta_{0}, \Delta_{1}\right)$, is an oriented graph, where $\Delta_{0}$ denotes the set of vertices, and $\Delta_{1}$ the set of arrows between the vertices. The origin and terminus of an arrow $a \in \Delta_{1}$, is denoted by $o(a)$ and $t(a)$ respectively.

We shall deal with finite connected quivers, that is the sets $\Delta_{0}$ and $\Delta_{1}$ are finite and the undirected graph will be connected. A path $\alpha$ in $\Delta$, is an ordered sequence of arrows, $\alpha=a_{1} \cdots a_{n}$, $a_{i} \in \Delta_{1}$ with $t\left(a_{i}\right)=o\left(a_{i+1}\right)$ for $i=1, \cdots, n-1$. We shall write $o(\alpha)=o\left(a_{1}\right)$ and $t(\alpha)=t\left(a_{n}\right)$ for the initial and terminal vertices of $\alpha$ respectively. An oriented cycle in $\Delta$, is a path $\alpha$, where $o(\alpha)=t(\alpha)$. The length or degree of $\alpha$, denoted $|\alpha|$ is equal to the number of arrows in $\alpha$ and the set of all paths of length $n$, is denoted $\Delta_{n}$. A vertex $e \in \Delta_{0}$ is considered to be a path of length zero with $o(e)=t(e)=e$. We shall allow $\Delta$ to have oriented cycles and multiple arrows between vertices.

Now we would like to make a semigroup out of the paths, and we will do this by first defining the set $\hat{\Delta}$ by

$$
\hat{\Delta}=\{\perp\} \cup \bigcup_{i=0}^{\infty} \Delta_{i}
$$

The multiplication in $\hat{\Delta}$ is, for $\gamma \in \Delta_{i}, \delta \in \Delta_{j}$, defined by $\gamma \cdot \delta=\gamma \delta$, if $t(\gamma)=o(\delta)$ and $\gamma \cdot \delta=\perp$ otherwise. For all $\alpha \in \hat{\Delta}$ we have $\alpha \cdot \perp=\perp \cdot \alpha=\perp$. For $k$ a commutative ring, we may now form the semigroup-algebra $k \hat{\Delta}$, and then we may view $k \Delta$, the path or quiver algebra on $\Delta$, as the quotient algebra $k \hat{\Delta} /(\perp)$. The benefit of this definition is that $k \Delta$ becomes a $\hat{\Delta}$-graded algebra. There is also an $\mathbb{N}$-grading on $k \Delta$, where $\operatorname{deg}_{\mathbb{N}} \alpha=n$, if $\alpha \in \Delta_{n}$. The paths of length $0, \Delta_{0}$ generate a subalgebra $k \Delta_{0}$ of $k \Delta$; hence $k \Delta$ is a $k \Delta_{0}$-bimodule. The identity element in $k \Delta$ is given by the sum of vertices. The class of algebras studied here are quotients of path algebras.

Definition 2. A quadratic monomial algebra $A$ is a quotient of a quiver algebra $k \Delta, A=k \Delta / I$, where $I=\left(\alpha_{1}, \ldots, \alpha_{n}\right)$ is a two sided homogeneous ideal generated by a set of paths of length two in $\Delta$.

Since $I$ is a two sided homogeneous ideal with respect to the standard grading, $A$ is a graded algebra and we may describe a 
canonical $k$-basis for $A$ and denote it $B(A)$. Such a basis consists of all paths that do not contain a path from $I$. A typical basis element in $A$ may be written as $a_{1} \ldots a_{n}$, where $a_{i} a_{i+1} \notin I$ for $1 \leq i \leq n-1$.

Example 1. Let $k$ be any field and $\Delta$ the following quiver:

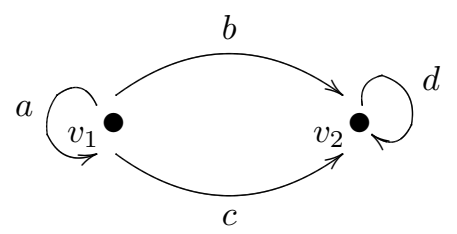

We may construct a quadratic monomial algebra which we shall denote by $A$ by choosing an ideal $I$, generated by paths of length two and then forming the quotient $A=k \Delta / I$. For instance if we let $I=(a a, d d)$ then the following is an example of multiplication in $A$ :

$$
v_{1} \cdot v_{1}=v_{1}, v_{1} \cdot v_{2}=0, v_{1} \cdot b=b, a \cdot b=a b, b \cdot a=0, \quad \text { etc. }
$$

The products

$$
a \cdot a=a a, \quad d \cdot d=d d
$$

are equal to 0 in $A$, since both of these paths are contained in $I$.

\section{PROJECTIVE MODULES AND PROJECTIVE RESOLUTIONS}

A central theme in the world of homological algebra is the exploration of the structure of rings and modules. Projective modules are a basic tool which are used extensively in this examination, since any module may be viewed as an epimorphic image of a projective module - just choose a set of generators $\left\{g_{i}\right\}$ for $M$ and map a projective module on a corresponding set of generators $\left\{e_{i}\right\}$ to $M$ by sending $e_{i}$ to $g_{i}$. In this way it is easy to compare any module to a projective module: if $d: P \longrightarrow M$ is an epimorphism, then we may say that $P$ differs from $M$ by the kernel of $d$. For more on this, see for example [3]. We will explore this idea in the present section. We will assume that we are working with left modules unless stated otherwise. There are several equivalent definitions of a projective module but the one that will be of most interest to us at present will be the following: 
Definition 3. Let $A$ be a ring. An $A$ module $P$ is projective if it is isomorphic to a direct summand of a free $A$ module.

We will now introduce the following notation with the quadratic monomial algebra $A$ of example 1 in mind.

Let

$P_{v_{1}}:=\left\langle\right.$ All paths in $A$ starting at $v_{1}$, including the zero path $\left.v_{1}\right\rangle$ $P_{v_{2}}:=\left\langle\right.$ All paths in $A$ starting at $v_{2}$, including the zero path $\left.v_{2}\right\rangle$ $P_{v_{1}} \cdot J:=\left\langle\right.$ All paths in $A$ starting at $v_{1}$ of length $\left.\geq 1\right\rangle$.

Since a path may only begin at either vertex $v_{1}$ or $v_{2}$, we have

$$
P_{v_{1}}+P_{v_{2}}=A \quad \text { and } \quad P_{v_{1}} \bigcap P_{v_{2}}=0
$$

and so

$$
A=P_{v_{1}} \oplus P_{v_{2}}
$$

$P_{v_{1}}$ and $P_{v_{2}}$ are direct summands of the left module $A$ (which is free over itself) and so we may regard them as (indecomposable) projective left $A$ modules. In the following we write im $d$ to denote the image of the homomorphism $d$ and ker $d$ to denote the kernel of $d$.

Definition 4. Let $M$ be an $A$-module. The sequence $P_{*}$ of $A$ modules and $A$-module homomorphisms

$$
P_{*}: \quad \cdots \stackrel{d_{3}}{\longrightarrow} P_{2} \stackrel{d_{2}}{\longrightarrow} P_{1} \stackrel{d_{1}}{\longrightarrow} P_{0} \stackrel{\varepsilon}{\longrightarrow} M \longrightarrow 0
$$

is called a complex if im $d_{i+1} \subseteq$ ker $d_{i}$ for each $i . P_{*}$ is said to be exact if the $\operatorname{im} d_{i+1}=\operatorname{ker} d_{i}$ for each $i . P_{*}$ is called a projective resolution of $M$ over $A$, if it is exact for all $i \geq 0$ and each $P_{i}$ is a projective $A$-module.

For now, $P_{*}$ will denote a projective resolution of $M$ and when we write deleted projective resolution, we shall mean $P_{*}$ with the $M$ term removed. We will be mainly interested in the case when $M=$ $A$. Projective resolutions are utilised in homological algebra as a way of approximating a module by using "well behaved" projective modules. It is well known that any $A$-module admits a projective resolution, see for instance [3] and we illustrate this below for the 
$A$-module $P_{v_{1}} / P_{v_{1}} \cdot J$ taking the quadratic monomial algebra $A$ in example 1 on page 41 , as a demonstration.

We begin by considering the epimorphism $\varepsilon$ in the following exact sequence:

$$
P_{v_{1}} \stackrel{\varepsilon}{\longrightarrow} P_{v_{1}} / P_{v_{1}} \cdot J \longrightarrow 0
$$

$P_{v_{1}}$ differs from $P_{v_{1}} / P_{v_{1}} \cdot J$ and this difference is recorded in ker $\varepsilon$.

We may now form the exact sequence:

$$
\operatorname{ker} \varepsilon \hookrightarrow P_{v_{1}} \stackrel{\varepsilon}{\longrightarrow} P_{v_{1}} / P_{v_{1}} \cdot J \longrightarrow 0
$$

but in the above, $\operatorname{ker} \varepsilon=P_{v_{1}} \cdot J$ is not projective as an $A$-module, since it is not a direct summand of the free module $A$. We may correct this blemish however by finding a projective resolution of $P_{v_{1}} \cdot J$. Consider the following sequence

$$
\text { ker } d_{1} \hookrightarrow P \stackrel{d_{1}}{\longrightarrow} P_{v_{1}} \cdot J \longrightarrow 0
$$

We would now like to construct a projective module $P$ and epimorphism $d_{1}$, making the aforementioned sequence exact. Note any path in $P_{v_{1}} \cdot J$ can be expressed using the generating set $\left\{v_{1} a \alpha, v_{1} b \beta, v_{1} c \gamma\right\}$, where $\alpha \in P_{v_{1}}, \beta \in P_{v_{2}}$, and $\gamma \in P_{v_{2}}$ (see quiver on page 41). If we replace $P$ with the direct sum of projective modules $P_{v_{1}} \oplus P_{v_{2}} \oplus P_{v_{2}}$ (which again results in a projective module), then a typical element in $P_{v_{1}} \oplus P_{v_{2}} \oplus P_{v_{2}}$ is of the form

$$
v_{1} \alpha+v_{2} \beta+v_{2} \gamma
$$

and we may define a surjective homomorphism $d_{1}$ on a generating element as follows:

$$
\begin{aligned}
d_{1}\left(v_{1} \alpha+v_{2} \beta+v_{2} \gamma\right) & =d_{1}\left(v_{1} \alpha\right)+d_{1}\left(v_{2} \beta\right)+d_{1}\left(v_{2} \gamma\right) \\
& =v_{1} a \alpha+v_{1} b \beta+v_{1} c \gamma
\end{aligned}
$$

It is easy to see that $d_{1}$ is surjective and $\operatorname{im} d_{1}=\operatorname{ker} \varepsilon=P_{v_{1}} \cdot J$ and so replacing ker $\varepsilon$, with $P_{v_{1}} \oplus P_{v_{2}} \oplus P_{v_{2}}$ in (1), the projective resolution of $P_{v_{1}} / P_{v_{1}} \cdot J$ now becomes:

$$
\text { ker } d_{1} \hookrightarrow P_{v_{1}} \oplus P_{v_{2}} \oplus P_{v_{2}} \stackrel{d_{1}}{\longrightarrow} P_{v_{1}} \stackrel{\varepsilon}{\longrightarrow} P_{v_{1}} / P_{v_{1}} \cdot J \longrightarrow 0
$$

Again as before, the ker $d_{1}$ is not projective as an $A$-module. We may again tackle this situation using the same approach as previously: forming a projective resolution of ker $d_{1}$. The kernel of $d_{1}$ 
is generated by $v_{1} \cdot a$. We need to define a homomorphism and projective module that maps onto ker $d_{1}$ :

$$
P_{v_{1}} \stackrel{d_{2}}{\longrightarrow} \operatorname{ker} d_{1} \longrightarrow 0 \quad \text { with } \quad v_{1} \stackrel{d_{2}}{\longrightarrow} v_{1} a
$$

and now the projective resolution of $P_{v_{1}} / P_{v_{1}} \cdot J$ takes the form:

$$
\text { ‥ } \stackrel{d_{3}}{\longrightarrow} P_{v_{1}} \stackrel{d_{2}}{\longrightarrow} P_{v_{1}} \oplus P_{v_{2}} \oplus P_{v_{2}} \stackrel{d_{1}}{\longrightarrow} P_{v_{1}} \stackrel{\varepsilon}{\longrightarrow} P_{v_{1}} / P_{v_{1}} \cdot J \longrightarrow 0
$$

If we delete $P_{v_{1}} / P_{v_{1}} \cdot J$ from the above exact complex, we get a projective resolution $P_{v_{1}} / P_{v_{1}} \cdot J$. We may view this deleted resolution as an approximation of the simple module $P_{v_{1}} / P_{v_{1}} \cdot J$.

\section{HochSCHILD COHOMOLOGY}

The appropriate cohomology theory for the class of associative $k$-algebras was first described by Gerhard Hochschild in [6]. Before continuing any further, we shall introduce the following notation: Given an arbitrary associative $k$-algebra $A$ with unit, we shall write $A^{e}=A \otimes_{k} A^{o p}$ to denote the enveloping algebra of $A$. Here we write $A^{o p}$ to denote the opposite algebra of $A$; as vector spaces $A$ and $A^{o p}$ are isomorphic but $A^{o p}$ is endowed with the opposite multiplication of $A$ :

$$
a^{o p} b^{o p}=(b a)^{o p}, \text { where } a, b \in A, \quad a^{o p}, b^{o p} \in A^{o p}
$$

By $\operatorname{Hom}_{A^{e}}(M, N)$ we shall mean the set of all $A^{e}$-homomorphisms from $M$ to $N$. This set may be endowed with the structure of an abelian group, where for $f, g \in \operatorname{Hom}_{A^{e}}(M, N)$ it may be shown that $f+g$ defined by $(f+g)(m)=f(m)+g(m)$ is an $A^{e}$-homomorphism for all $m \in M$.

$\operatorname{Ext}_{A^{e}}(A, M)$ may be defined as the Hochschild cohomology group $H^{*}(A, M)$ of $A$ with coefficients in the $A$-bimodule $M$. When $M=A$, it may be shown that $\operatorname{Ext}_{A^{e}}(A, A)$ also possesses a rich multiplicative structure:

$$
\operatorname{Ext}_{A^{e}}^{m}(A, A) \otimes_{k} \operatorname{Ext}_{A^{e}}^{n}(A, A) \longrightarrow \operatorname{Ext}_{A^{e}}^{m+n}(A, A)
$$

turning $\operatorname{Ext}_{A^{e}}(A, A)$ into a graded commutative algebra. An algebra $A$ is graded commutative (or supercommutative) with homogeneous elements $a$ and $b$ of degree $m$ and $n$ in $A$ respectively if

$$
a b=(-1)^{m n} b a \text {. }
$$


To compute the aforementioned cohomology groups of $A$, we begin by first applying the left exact functor $\operatorname{Hom}_{A^{e}}(\cdot, M)$ to the deleted standard projective resolution of $A$, where the $n$th projective module has the form $P_{n}=A \otimes_{k} A^{\otimes n} \otimes_{k} A$ and so the resolution may be written:

$$
\cdots \longrightarrow A \otimes A^{\otimes n} \otimes A \stackrel{d_{n}}{\longrightarrow} \cdots \stackrel{d_{2}}{\longrightarrow} A \otimes A \otimes A \stackrel{d_{1}}{\longrightarrow} A \otimes A \longrightarrow 0
$$

The differential $d_{n}$ is given by

$$
\begin{aligned}
d_{n}\left(a_{0} \otimes a_{1} \cdots a_{n} \otimes a_{n+1}\right) & =a_{0} a_{1} \otimes a_{2} \cdots a_{n} \otimes a_{n+1} \\
& +\sum_{i=1}^{n-1}(-1)^{i} a_{0} \otimes a_{1} \cdots\left(a_{i} a_{i+1}\right) \cdots a_{n} \otimes a_{n+1} \\
& +(-1)^{n} a_{0} \otimes a_{1} \cdots a_{n-1} \otimes a_{n} a_{n+1}
\end{aligned}
$$

Now using the isomorphism $\operatorname{Hom}_{A^{e}}\left(A \otimes A^{\otimes n} \otimes A, M\right) \cong \operatorname{Hom}_{k}\left(A^{\otimes n}, M\right)$, we get the so called Hochschild complex:

$$
0 \longrightarrow A \stackrel{\delta^{0}}{\longrightarrow} \operatorname{Hom}_{k}(A, M) \stackrel{\delta^{1}}{\longrightarrow} \cdots \stackrel{\delta}{\longrightarrow} \operatorname{Hom}_{k}\left(A^{\otimes n}, M\right) \stackrel{\delta}{\longrightarrow} \cdots
$$

The $n$th Hochschild cohomology module of $A$ with coefficients in $M$ is given by

$$
H^{n}(A, M) \cong \operatorname{ker} \delta^{n} / \operatorname{im} \delta^{n-1} \cong \operatorname{Ext}_{A^{e}}(A, M) .
$$

In particular, we shall be interested in the case when $M=A$ and we shall write $H H^{*}(A)$ instead of $H^{*}(A, A)$. In this instance the differential $\delta$ has the following form:

$$
\delta^{0}: A \longrightarrow \operatorname{Hom}_{k}(A, A) \quad \text { with } \quad\left(\delta^{0} b\right)(a)=a b-b a \quad \text { for } a, b \in A .
$$

and for $n \geq 1, \quad \delta^{n}: \operatorname{Hom}_{k}\left(A^{\otimes n}, A\right) \longrightarrow \operatorname{Hom}_{k}\left(A^{\otimes(n+1)}, A\right)$;

$$
\begin{aligned}
\left(\delta^{n} f\right)\left(a_{1} \otimes \cdots a_{n+1}\right) & =a_{1} f\left(a_{2} \otimes \cdots \otimes a_{n+1}\right) \\
& +\sum_{1 \leq j \leq n}(-1)^{j} f\left(a_{1} \otimes \cdots \otimes a_{j} a_{j+1} \otimes \cdots \otimes a_{n+1}\right) \\
& +(-1)^{n+1} f\left(a_{1} \otimes \cdots \otimes a_{n}\right) a_{n+1}
\end{aligned}
$$


4.1. Interpreting the 0 th and 1st cohomology groups. In this subsection we highlight some aspects of the cohomology groups in dimensions $\leq 2$. In particular the Hochschild cohomology groups may be interpreted as providing an insight into the structure of an algebra. We begin by considering $H H^{0}(A)$. This group is isomorphic to the kernel of $\delta^{0}$ and hence consists of all those elements in $A$ that commute with all elements in $A$, that is $H H^{0}(A)$ is the centre of $A$ :

$$
H H^{0}(A) \cong\{b \in A \mid a b=b a \text { for all } a \in A\} .
$$

Definition 5. A derivation of $A$ to $A$ is an $k$-module homomorphism $f: A \longrightarrow A$ that satisfies Leibnitz's rule:

$$
f(a b)=a f(b)+f(a) b \quad \text { for all } a, b \in A
$$

A derivation $f$ of $A$ to $A$ is an inner derivation if there exists $b \in A$ such that:

$$
f(a)=a b-b a \quad \text { for all } a \in A
$$

Now returning to the coboundary $\delta$ and setting

$$
\delta^{1}(f)(a \otimes b)=a f(b)-f(a b)+f(a) b
$$

equal to zero, we observe that a 1-cocycle (an element in the kernel of $\delta^{1}$ ) is also a linear map $f: A \longrightarrow A$ which satisfies Leibnitz's condition. Similarly for each $b \in A$,

$$
\delta^{0}(b)(a)=a b-b a \quad \text { for all } a \in A
$$

and so there is a one-to-one correspondence between the coboundaries lying in $\operatorname{im} \delta^{0}$ and the inner derivations of $A$. The $k$-module $H H^{1}(A)$ may be interpreted as the space of all bimodule derivations of $A$ modulo the inner derivations of $A$.

There are also connections to algebraic geometry. In [4], Murray Gerstenhaber introduced a deformation theory for rings and algebras based on formal power series. A formal deformation of an associative algebra $(A, \mu)$ is an associative algebra $A[[t]]$ with a multiplication $\mu_{t}$ defined by

$$
\mu_{t}(p, q)=\mu(p, q)+t \mu_{1}(p, q)+t^{2} \mu_{2}(p, q)+\cdots
$$

where $p, q \in A$. The algebra is said to be rigid if every formal deformation is isomorphic to a trivial deformation. One may show that separable semi-simple algebras are rigid. In the same paper 
Gerstenhaber observed that algebras $A$ which satisfy $H H^{2}(A)=0$ are rigid.

\subsection{A Projective Resolution of a Quadratic Monomial Al-}

gebra. In general a module may have several projective resolutions. When one wishes to compute (co)homology, a minimal projective resolution is best. In the following we shall state a minimal projective $A^{e}$ - resolution for a quadratic monomial algebra. This minimal projective resolution was constructed by Emil Sköldberg in[8]. In order to write down this resolution the following definition will be required:

Definition 6. Let $A=k \Delta / I$ be an algebra such that $I$ is an ideal generated by quadratic monomials. Define the ideal $J$ to be generated by all quadratic monomials that do not lie in $I$; then the Koszul dual of $A$, denoted by $A^{!}$, is defined by $A^{!}=k \Delta / J$.

Example 2. For the given quadratic monomial algebra $A$ in example 1 on page 41 , we have $A^{!}=k \Delta / J$, with $J=(a b, a c, c d, b d) . A^{!}$ has the same generators of $A$, with the following as an example of multiplication in $A^{!}$:

$$
v_{1} \cdot v_{1}=v_{1}, v_{1} \cdot v_{2}=0, v_{1} \cdot b=b, a \cdot a=a a, d \cdot d=d d, \quad \text { etc. }
$$

The products

$$
a \cdot b=a b, \quad d \cdot d=d d \quad a \cdot c=a c, \quad c \cdot d=c d
$$

are equal to zero in $A^{!}$, since all products are contained in $J$.

The projective modules in the minimal projective graded $A^{e}$-resolution of $A$ have the following description. From here on, when we write $P_{*}$ we shall be referring to the following minimal $A^{e}$-resolution.

Lemma 1. If $A=k \Delta / I$ is a quadratic monomial algebra, then a minimal projective resolution of $A$ given as a left $A^{e}$-module is

$$
P_{i}=A \otimes_{k \Delta_{0}} A_{i}^{!} \otimes_{k \Delta_{0}} A
$$

and the $A^{e}$-linear differential is defined on the basis elements by $d_{i}\left(1 \otimes a_{1} \cdots a_{i} \otimes 1\right)=a_{1} \otimes a_{2} \cdots a_{i} \otimes 1+(-1)^{i} 1 \otimes a_{1} \cdots a_{i-1} \otimes a_{i}$ Proof. See [8]. 
Example 3. Consider $A=k \Delta / I$, where

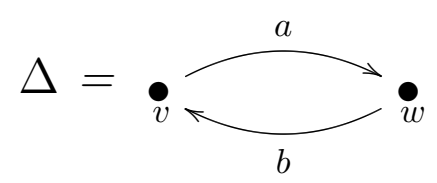

$I=(a b, b a)$ and $J=(\emptyset)$. The projective modules $P_{i}$ in $P_{*}$, have the form, where we write $\otimes=\otimes_{k \Delta_{0}}$ :

$$
\begin{aligned}
& A \otimes a b a \otimes A \quad A \otimes a b \otimes A \quad A \otimes a \otimes A \quad A \otimes v \otimes A \\
& \text {.. } \stackrel{d_{4}}{\longrightarrow} \oplus \stackrel{d_{3}}{\longrightarrow} \oplus \stackrel{d_{2}}{\longrightarrow} \oplus \stackrel{d_{1}}{\longrightarrow} \oplus \quad \stackrel{\varepsilon}{\longrightarrow} A \rightarrow 0 \\
& A \otimes b a b \otimes A \quad A \otimes b a \otimes A \quad A \otimes b \otimes A \quad A \otimes w \otimes A
\end{aligned}
$$

Notice that the module $P_{0}$ records the vertices of the quiver, $P_{1}$ records the arrows that generate $A, P_{2}$ records the relations of $A$, $P_{3}$ records the relations $(a b) a=a(b a)$ among relations of $A$, etc.

\section{Cohomology of a Quadratic Monomial Algebra}

In a forthcoming paper with Emil Sköldberg, the cohomology groups for a quadratic monomial algebra are explicitly described. In this section we illustrate this theory by calculating the Hochschild cohomology groups of the given quadratic monomial algebra $A$ in example 3 on page 48 . We begin with the following complex:

$$
0 \longrightarrow \operatorname{Hom}_{A^{e}}\left(P_{0}, A\right) \stackrel{\delta^{0}}{\longrightarrow} \cdots \stackrel{\delta^{n-1}}{\longrightarrow} \operatorname{Hom}_{A^{e}}\left(P_{n}, A\right) \stackrel{\delta^{n}}{\longrightarrow} \cdots
$$

The coboundary $\delta$ is induced by the differential $d$ on $P_{*}$ :

$$
\begin{aligned}
P_{i+1} \stackrel{d_{i+1}}{\delta^{i+1}(f)} & \\
\delta^{i+1} f\left(1 \otimes a_{1} \cdots a_{i+1} \otimes 1\right) & =f\left(d_{i+1}\left(1 \otimes a_{1} \cdots a_{i+1} \otimes 1\right)\right) \\
& =f\left(a_{1} \otimes a_{2} \cdots a_{i+1} \otimes 1\right) \\
& +(-1)^{i+1} f\left(1 \otimes a_{1} \cdots a_{i} \otimes a_{i+1}\right)
\end{aligned}
$$

where $f \in \operatorname{Hom}_{A^{e}}\left(P_{i}, A\right)$. As we have seen earlier, the $n$th Hochschild cohomology module of $A$ with coefficents in $A$, may be found by computing

$$
H H^{n}(A) \cong \operatorname{ker} \delta^{n} / \operatorname{im} \delta^{n-1} \cong \operatorname{Ext}_{A^{e}}^{n}(A, A)
$$


We will use the following lemma to simplify our calculation of the cohomology groups.

Lemma 2. The map $\phi: \operatorname{Hom}_{A^{e}}\left(P_{i}, A\right) \longrightarrow \operatorname{Hom}_{k \Delta_{0}^{e}}\left(A_{i}^{!}, A\right)$ defined by

$$
\phi(f)\left(a_{1} \cdots a_{i}\right):=f\left(1 \otimes_{k \Delta_{0}} a_{1} \cdots a_{i} \otimes_{k \Delta_{0}} 1\right),
$$

$f \in \operatorname{Hom}_{A^{e}}\left(P_{i}, A\right)$ is a chain map and vector space isomorphism for each $i$.

Proof. This result is proved in the authors thesis.

We have established through lemma 2 that calculating the cohomology of the cochain complex at (2), will yield the same results as computing the cohomology of the following cochain complex:

$$
0 \longrightarrow \operatorname{Hom}_{k \Delta_{0}^{e}}\left(A_{0}^{!}, A\right) \stackrel{\bar{\delta}^{0}}{\longrightarrow} \cdots \stackrel{\bar{\delta}^{n-1}}{\longrightarrow} \operatorname{Hom}_{k \Delta_{0}^{e}}\left(A_{n}^{!}, A\right) \stackrel{\bar{\delta}^{n}}{\longrightarrow} \cdots
$$

We shall now take a moment to describe the coboundary homomorphism $\bar{\delta}$ and the $k$-module $\operatorname{Hom}_{k \Delta_{0}^{e}}\left(A^{!}, A\right)$ in a little more detail. An element $f \in \operatorname{Hom}_{k \Delta_{0}^{e}}\left(A^{!}, A\right)$ is a $k \Delta_{0}^{e}$ - linear homomorphism from $A^{!}$to $A$. For $\alpha \in B\left(A^{!}\right)$and $\beta \in B(A)$, we shall use the notation $(\alpha, \beta)$ for the morphism $\alpha \stackrel{f}{\longmapsto} \beta$, and $\gamma \mapsto 0$ for all other basis elements $\gamma \in B\left(A^{!}\right)$. We shall write $o(\alpha)=v$ and $t(\alpha)=w$. Since $f \in \operatorname{Hom}_{k \Delta_{0}^{e}}\left(A_{i}^{!}, A\right)$ is linear over the vertices, we have

$$
f(\alpha)=f(v \cdot \alpha)=v \cdot f(\alpha)=v \cdot \beta
$$

and

$$
f(\alpha)=f(\alpha \cdot w)=f(\alpha) \cdot w=\beta \cdot w
$$

and so for an $f \in \operatorname{Hom}_{k \Delta_{0}^{e}}\left(A^{!}, A\right)$, we shall write $(\alpha, \beta)$ such that $o(\alpha)=o(\beta)$ and $t(\alpha)=t(\beta)$. It is shown in [7] that the coboundary operator $\bar{\delta}$ on these basis elements is given by

$$
\bar{\delta}(\alpha, \beta)=\sum_{a \in \Delta_{1}}(a \alpha, a \beta)+(-1)^{|\alpha|+1} \sum_{b \in \Delta_{1}}(\alpha b, \beta b)
$$

From here on we shall write $\delta$ in of place of $\bar{\delta}$. We are now in a position to calculate the cohomology groups of the quadratic monomial algebra given in example 2, page 48. 
Example 4. We begin with the following deleted projective resolution of $A$ :

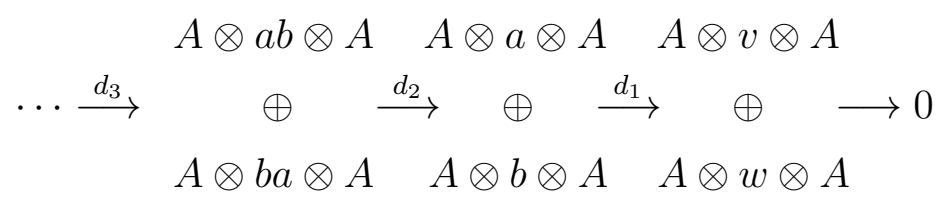

Applying $\operatorname{Hom}_{A^{e}}(\cdot, A)$ and then using the isomorphism $\operatorname{Hom}_{A^{e}}\left(P_{i}, A\right) \cong$ $\operatorname{Hom}_{k \Delta_{0}^{e}}\left(A_{i}^{!}, A\right)$,we get the resulting cochain complex :

$$
\begin{aligned}
& \operatorname{Hom}_{k \Delta_{0}^{e}}(v, A) \quad \operatorname{Hom}_{k \Delta_{0}^{e}}(a, A) \quad \operatorname{Hom}_{k \Delta_{0}^{e}}(a b, A)
\end{aligned}
$$

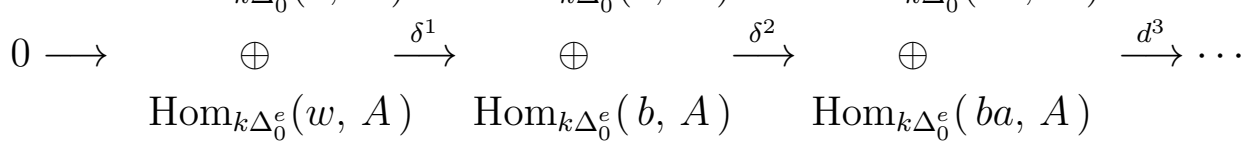

We would now like to calculate the cohomology in each degree of this complex but before doing this we note a $k$-basis for $\operatorname{Hom}_{k \Delta_{0}^{e}}\left(A^{!}, A\right)$ is given by all $(\alpha, \beta)$ with $o(\alpha)=o(\beta)$ and $t(\alpha)=t(\beta)$. Hence we may simplify the notation in the aforementioned complex by rewriting it as:

$$
\begin{array}{ccc}
k \cdot(v, v) & k \cdot(a, a) & k \cdot(a b, v) \\
0 \longrightarrow & \oplus \stackrel{\delta^{0}}{\longrightarrow} \underset{k}{\longrightarrow} \stackrel{\oplus}{\longrightarrow} \underset{\delta^{1}}{\longrightarrow} \stackrel{\delta^{2}}{\longrightarrow} \cdots
\end{array}
$$

We may now calculate the cohomology groups in each degree. We begin by computing $H H^{0}(A) \cong \operatorname{ker} \delta^{0}$ :

$$
\begin{aligned}
\delta^{0}(\lambda(v, v)+\mu(w, w)) & =\lambda((b, b)-(a, a)) \\
& +\mu((a, a)-(b, b))
\end{aligned}
$$

which is equal to zero $\Leftrightarrow \lambda=\mu$ for $\lambda, \mu \in k$.

Hence the kernel of $\delta^{0}$ is one dimensional and is generated by $(u, u)+(v, v)$ and so $H H^{0}(A) \cong k$.

Next we compute $H H^{1}(A)$. Since $\operatorname{dim}\left(\operatorname{ker} \delta^{0}\right) \cong k$ and $\delta^{0}$ is a map from a two dimensional vector space, we have $\operatorname{dim}\left(\operatorname{im} \delta^{0}\right) \cong k$. Now

$$
\delta^{1}(\lambda(a, a))=\lambda(b a, b a)+\lambda(a b, a b)=0
$$

since $b a \in J$ or $b a \in I$ and $a b \in J$ or $a b \in I$. For the same reason we also have

$$
\delta^{1}((\mu(b, b)))=\mu(a b, a b)+\mu(b a, b a)=0
$$

Hence $\operatorname{ker} \delta^{1} \cong k \oplus k$ and so we have $H H^{1}(A) \cong k$. 
Finally we compute $H H^{2}(A)$. Since the kernel of $\delta^{1}$ is two dimensional, this means the dimension of the image $\operatorname{im} \delta^{1}$ is trivial. Computing as before, it is easy to show that the kernel of $\delta^{2}$ is generated by $(a b, v)+(b a, w)$ and so $\operatorname{dim}\left(\operatorname{ker} \delta^{2}\right) \cong k$. Hence $H H^{2}(A) \cong k$.

6. Interpreting the 0th \& 1st cohomology groups of $A$

As we have already seen, the zeroth cohomology group $H H^{0}(A)$ coincides with the centre of $A$. We shall illustrate this with an example.

Example 5. Let $A=k \Delta / I$ be the quadratic monomial algebra obtained from the quiver

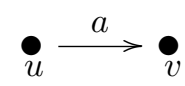

where in this instance $I=J=\{\emptyset\}$. Note the centre of an algebra $A$ consists of all those $x$ of $A$ such that $x a=a x$ for all $a$ in $A$.

We shall first compute the centre of the given quadratic monomial algebra:

$$
\begin{aligned}
(\lambda u+\varphi v+\psi a) \cdot u & =u \cdot(\lambda u+\varphi v+\psi a) \\
& \Leftrightarrow \lambda u=\lambda u+\psi a \Rightarrow \psi=0 \\
(\lambda u+\varphi v) \cdot a & =a \cdot(\lambda u+\varphi v) \\
& \Leftrightarrow \lambda a=\varphi a \Rightarrow \lambda=\varphi
\end{aligned}
$$

and so the centre of $A$ is a one dimensional vector space spanned by $\langle u+v\rangle$. Computing $H H^{0}(A)$ from the following complex:

$$
0 \longrightarrow k \cdot(u, u) \oplus k \cdot(v, v) \longrightarrow k \cdot(a, a) \longrightarrow 0
$$

it is easy to show that it is a one dimensional vector space generated by $(u, u)+(v, v)$. Hence the centre of $A$ and $H H^{0}(A)$ are isomorphic as vector spaces.

Example 6. Let $A=k \Delta / I$ be obtained from the following quiver

$$
\Delta:=\quad \text { e } \stackrel{a}{\longrightarrow} \text { i }
$$

We write $f \in \operatorname{Hom}_{k}(A, A)$ as 


$$
\begin{aligned}
& f(u)=\lambda_{1} u+\lambda_{2} v+\lambda_{3} a+\lambda_{4} b \\
& f(v)=\tau_{1} u+\tau_{2} v+\tau_{3} a+\tau_{4} b \\
& f(a)=\varphi_{1} u+\varphi_{2} v+\varphi_{3} a+\varphi_{4} b \\
& f(b)=\psi_{1} u+\psi_{2} v+\psi_{3} a+\psi_{4} b
\end{aligned}
$$

for all $\lambda_{i}, \tau_{i}, \varphi_{i}, \psi_{i} \in k$. The derivations of $A$ are defined as those $k$-module homomorphisms $f: A \longrightarrow A$ that satisfy

$$
f(a b)=a f(b)+f(a) b, \quad \text { for all } a, b \in A .
$$

We shall denote this group of derivations by $\operatorname{Der}(A, A)$ and we begin now by computing all the derivations of the given algebra $A$ :

$$
\begin{aligned}
\lambda_{1} u+\lambda_{2} v+\lambda_{3} a+\lambda_{4} b=f(u) & =f(u \cdot u)=u \cdot f(u)+f(u) \cdot u \\
& =2 \lambda_{1} u+\lambda_{3} a+\lambda_{4} b
\end{aligned}
$$

This implies $\lambda_{1} u-\lambda_{2} v=0$ or $\lambda_{1}=\lambda_{2}=0$.

$0=f(u \cdot v)=u \cdot f(v)+f(u) \cdot v=\tau_{1} u+\tau_{3} a+\tau_{4} b+\lambda_{2} v+\lambda_{3} a+\lambda_{4} b$

This means $\tau_{3}=-\lambda_{3}, \tau_{4}=-\lambda_{4}, \tau_{1}=0, \lambda_{2}=0$. Continuing in the same way and computing the remaining 14 derivations:

$$
\begin{gathered}
f(u \cdot a), f(u \cdot b), f(v \cdot u), f(v \cdot v), f(v \cdot a) f(v \cdot b), f(a \cdot a), \\
f(a \cdot v), f(a \cdot b), f(a \cdot u), f(b \cdot u), f(b \cdot a), f(b \cdot b), f(b \cdot v)
\end{gathered}
$$

we also have $\lambda_{1}=\tau_{2}=\psi_{1}=\psi_{2}=\varphi_{1}=\varphi_{2}=0$ Substituting $\tau_{3}=-\lambda_{3}, \tau_{4}=-\lambda_{4}$, the $k$-module of all derivations from $A$ to $A$ is spanned by:

$$
\begin{aligned}
& f(u)=\lambda_{3} a+\lambda_{4} b, \\
& f(v)=-\lambda_{3} a-\lambda_{4} b=-f(u), \\
& f(a)=\varphi_{3} a+\varphi_{4} b \\
& f(b)=\psi_{3} a+\psi_{4} b
\end{aligned}
$$

for $\lambda_{3}, \lambda_{4}, \varphi_{3}, \varphi_{4}, \psi_{3}, \psi_{4} \in k$. Hence $\operatorname{Der}(A, A)$ is a 6 -dimensional vector space, with basis, given by the set of all derivations $f_{i}: A \longrightarrow A, 1 \leq i \leq 6$;

$$
f_{1}(x)=\left\{\begin{array}{rl}
a, & \text { if } x=u, \\
-a, & \text { if } x=v, \\
0 & \text { otherwise. }
\end{array} \quad f_{2}(x)=\left\{\begin{array}{cl}
b, & \text { if } x=u \\
-b, & \text { if } x=v \\
0 & \text { otherwise. }
\end{array}\right.\right.
$$




$$
\begin{aligned}
& f_{3}(x)=\left\{\begin{array}{ll}
a, & \text { if } x=a, \\
0 & \text { otherwise. }
\end{array} \quad f_{4}(x)= \begin{cases}b, & \text { if } x=a, \\
0 & \text { otherwise. }\end{cases} \right. \\
& f_{5}(x)=\left\{\begin{array}{ll}
a, & \text { if } x=b, \\
0 & \text { otherwise. }
\end{array} \quad f_{6}(x)= \begin{cases}b, & \text { if } x=b, \\
0 & \text { otherwise. }\end{cases} \right.
\end{aligned}
$$

where $x$ a basis element in $A$. The inner derivations of $A$ are computed next.

$$
\begin{array}{ll}
u \longrightarrow u \cdot u-u \cdot u=0 & u \longrightarrow u \cdot v-v \cdot u=0 \\
v \longrightarrow v \cdot u-u \cdot v=0 & v \longrightarrow v \cdot v-v \cdot v=0 \\
a \longrightarrow a \cdot u-u \cdot a=-a & a \longrightarrow a \cdot v-v \cdot a=a \\
b \longrightarrow b \cdot u-u \cdot b=-b & b \longrightarrow b \cdot v-v \cdot b=b \\
=-f_{3}-f_{6} & =f_{3}+f_{6}=-\left(-f_{3}-f_{6}\right) \\
u \longrightarrow u \cdot a-a \cdot u=a & u \longrightarrow u \cdot b-b \cdot u=b \\
v \longrightarrow v \cdot a-a \cdot v=-a & v \longrightarrow v \cdot b-b \cdot v=-b \\
a \longrightarrow a \cdot a-a \cdot a=0 & a \longrightarrow a \cdot b-b \cdot a=0 \\
b \longrightarrow b \cdot a-a \cdot b=0 & b \longrightarrow b \cdot b-b \cdot b=0 \\
=f_{1} & =f_{2}
\end{array}
$$

The inner derivations form a 3-dimensional subspace of $\operatorname{Der}(\mathrm{A}, \mathrm{A})$, and so the quotient space of derivations modulo inner derivations is:

\section{$\frac{k \oplus k \oplus k \oplus k \oplus k \oplus k}{k \oplus k \oplus k} \cong k \oplus k \oplus k$}

On the other hand, suppose we calculate the Hochschild cohomology of the complex associated with the given algebra:

$0 \longrightarrow k(u, u) \oplus k(v, v) \stackrel{\delta^{0}}{\longrightarrow} k(a, a) \oplus k(b, b) \oplus k(b, a) \oplus k(a, b) \stackrel{\delta^{1}}{\longrightarrow} 0$

we then have

$$
H H^{1}(A) \cong \frac{k \oplus k \oplus k \oplus k}{k} \cong k \oplus k \oplus k
$$

Hence as vector spaces, the space of outer derivations modulo the space of inner derivations and $H H^{1}(A)$ are isomorphic (as expected from section 4.1). 


\section{REFERENCES}

[1] Ames, Guillermo, Cagliero Leandro, Tirao Paulo, The cohomology ring of truncated quiver algebras, arXiv:math.KT/0603056v1

[2] Cibils, Claude, Hochschild cohomology algebra of radical square zero algebras, Algebras and modules, II (Geiranger, 1996), CMS Conf. Proc., vol.24, Amer.Math.Soc., Providence, RI, 1998, pp.93-101.

[3] Eisenbud David, Commutative Algebra with a View Toward Algebraic Geometry, Springer (1996).

[4] Gerstenhaber, M., On the deformations of rings and algebras, Ann. of Math. 79 (1964), 59-103.

[5] Happel, Dieter, Hochschild cohomology of finite dimensional algebras, Book Series lecture Notes in Mathematics publisher Springer Berlin (1989).

[6] Hochschild Gerhard, On the cohomology groups of an associative algebra, Ann. of Math. (2) Vol.46, No.1, (1945) 58-67.

[7] O'Keeffe David, The Hochschild Cohomology Ring of a Quadratic Monomial Algebra, Ph.D Thesis, NUI Galway (2009).

[8] Sköldberg Emil, The Hochschild homology of truncated and quadratic monomial algebras, J.London Math.Soc., 59 (1999), 76-86.

David O'Keeffe received his primary degree at National University of Ireland, Maynooth, in 1998, and his Ph. D. at National University of Ireland, Galway in 2009.

School of Mathematics, Statistics, and Applied Mathematics, NaTIONAL UNIVERSITY OF IRELAND GALWAY

E-mail address: david.okeeffe@nuigalway.ie 